\title{
Improvement of Hybrid EL Efficiency in Nanoparticle EL Devices by Insertion of the Layers of $\mathrm{PVK}$ and $\mathrm{BaF}_{2}$
}

\author{
Jun Woo Lee, Kyoungah Cho, Hyunsuk Kim, Byoungjun Park, and Sangsig Kim ${ }^{\mathrm{a}}$ \\ Department of Electrical Engineering and Institute for Nano Science, Korea University, \\ Anam-dong 5-ga, Seongbuk-gu, Seoul 136-701, Korea \\ Sung Hyun Kim \\ Department of Chemical and Biological Engineering, Korea University, \\ Anam-dong 5-ga, Seongbuk-gu, Seoul 136-701, Korea \\ ${ }^{a}$ E-mail : sangsig@korea.ac.kr
}

(Received January 27 2005, Accepted May 19 2005)

\begin{abstract}
Electroluminescence(EL) and current-voltage(I-V) characteristics of hybrid EL devices containing $\mathrm{Pr}$ and $\mathrm{Mn}$ co-doped $\mathrm{ZnS}$ nanoparticles were investigated in this study. For the insertion of a hole transport layer of poly (N-vinyl carbazole)(PVK), the current level became lower due to the accumulation of electrons at the interface between PVK and nanoparticles. When both PVK and buffer layer $\mathrm{BaF}_{2}$ were simultaneously introduced, the enhanced EL efficiency and improved I-V characteristics were obtained. This results from the additional increase of hole injection owing to the internal field induced by the significant accumulation of electrons at the interface. The presence of buffer layer $\mathrm{BaF}_{2}$ together with PVK makes it possible the charge accumulation enough to induce the sufficient internal field for further hole injection.
\end{abstract}

Keywords : Nanoparticles, Hybrid EL device, Electroluminescence

\section{INTRODUCTION}

Recently, much attention has been paid on the enhanced efficiency of hybrid electroluminescence(EL) devices which make use of nanoparticles as emitting materials[1]. Focus of studies is on two major subjects; introduction of various nanoparticles such as doped nanoparticles or core/shell nanoparticles, and optimization of EL device structures by introducing organic materials[1-3]. Semiconductor nanoparticles are regarded as high efficient materials due to the distinguishable properties such as size-dependence of electrical and optical properties and discrete distribution of the state density[4]. The hybrid EL devices take the following advantages of nanoparticles; high quantum yield, tunable emission colors, electron affinities, and ionization potentials adjusted by size variation of nanoparticles. The functional principles of the devices are the same as organic light emitting diodes(OLEDs) which are based on the injection of positive and negative carriers from electrodes, charge transport, formation of exciton, and radiative decay. Thus the charge balance inside the device is one of the most significant factors in the enhancement of hybrid EL efficiency. When the mobility of two carriers is the same through the device, the exact charge balance is reached and the maximum efficiency can be attained. However, semiconductor nanoparticles in general have large ionization potential leading to high hole injection barrier. Consequently, electrons are the dominant carriers inside the devices. To solve this disadvantage, hybrid EL devices of energystep-like structure have been studied through the introduction of hole injection layer(HIL) or hole transport layer(HTL) of organic materials. It has been well-known that the use of PEDOT:PSS and PVK as HIL and HTL in the Mn doped ZnS nanoparticles improve the current-voltage(I-V) characteristics[2]. For undoped nanoparticles, similar results were reported in Ref.5. Electron injection is influenced by the formation of space charge inside the nanoparticle layer due to the transport mechanism of charge carriers. So the cathode treatment using additional layer is necessary for the improved device yields.

In this work, we investigate EL as well as I-V characteristics of the devices which consist of different structures in point of the insertion of HTL and buffer layer between cathode and nanoparticles. The significant effect of the additional layers on the device performance 
was observed; enhanced EL efficiency and improved I-V characteristics. In this paper, the influences of HTL and buffer layers on device yields, especially charge balance, are compared with the single layer device. Finally, the mechanisms for the improvement of charge balance are explained.

\section{EXPERIMENTAL}

$\mathrm{ZnS}$ nanoparticles co-doped with $\mathrm{Mn}$ and $\mathrm{Pr}$ were prepared by a wet chemical method and dispersed in methanol[6]. Three hybrid EL devices under study are ITO/ZnS:(Pr,Mn) nanoparticles/Al, ITO/PVK/ZnS:(Pr,Mn) nanoparticles/Al, and TTO/PVK/ZnS:(Pr,Mn) nanoparticles/ $\mathrm{BaF}_{2} / \mathrm{Ca} / \mathrm{Al}$. These devices were fabricated as follows. First, a transparent and conductive substrate of indium tin oxide(ITO) glass was selectively etched in aqua regia to pattern the emission area and electrode. The patterned ITO glass was cleaned by sonication in acetone, isopropyl alcohol, and purified water. For the use of the hole transport layer, the $35 \mathrm{~nm}$-thick PVK $(0.17 \mathrm{~g}$ in 20 $\mathrm{ml}$ toluene) layer was spin-coated onto the patterned ITO glass. Then $\mathrm{ZnS}$ nanoparticles co-doped with $\mathrm{Mn}$ and $\mathrm{Pr}$ dispersed in methanol was spin-deposited on the PVK layer. For no use of HTL(PVK), nanoparticles were directly coated onto the patterned ITO glass. After deposition. of an emission layer(EML), the active area was defined by metal mask. To use a buffer layer, the 2 $\mathrm{nm}$-thick $\mathrm{BaF}_{2}$ was vacuum-evaporated onto the nanoparticle layer. Then $5 \mathrm{~nm}$-thick $\mathrm{Ca}$ and $150 \mathrm{~nm}$ thick $\mathrm{Al}$ metal layers were deposited by thermal evaporation to complete the devices. For no use of the buffer layer, the $150-\mathrm{nm}$ thick $\mathrm{Al}$ layer was thermally evaporated on the top of the nanoparticles without any Ca layer.

Figure 1 shows the schematic drawing of an EL device of ITO/ZnS:(Pr,Mn)nanoparticles/Al. At forward bias, the positive contact of voltage source was connected to the ITO electrode.

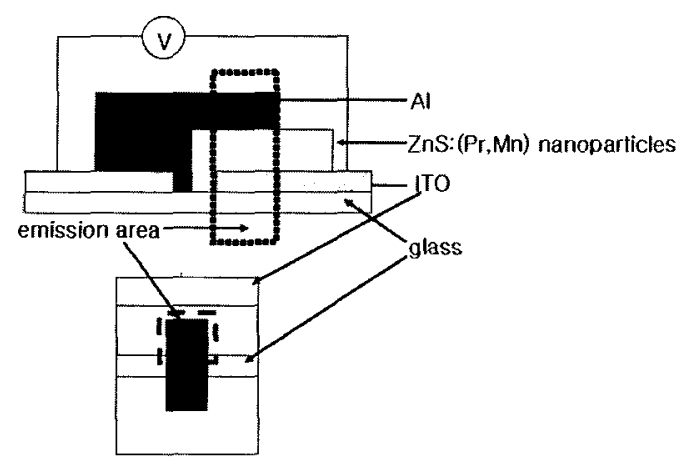

Fig. 1. Schematic drawing of an ITO/ZnS:(Pr,Mn) nanoparticles/Al device.
I-V characteristics of the prepared devices were obtained using Keithley 237 SMU. EL spectra was taken at room temperature using a single grating monochromator and detected by thermoelectrically cooled GaAs photomultiplier tube.

\section{RESULTS AND DISCUSSION}

EL spectra of ITO/ZnS:(Pr,Mn)nanoparticles/Al and ITO/PVK/ZnS:(Pr,Mn)nanoparticles $/ \mathrm{BaF}_{2} / \mathrm{Ca} / \mathrm{Al}$ devices taken in this study are compared in Fig. 2. The single layer EL device without HTL(PVK) and buffer layer $\left(\mathrm{BaF}_{2}\right)$ shows a broad EL band ranging from 530 to $620 \mathrm{~nm}$, but the device yield is lower than that of the multilayer device consisting of ITO/PVK/ZnS:(Pr,Mn) nanoparticles $/ \mathrm{BaF} / \mathrm{Ca} / \mathrm{Al}$.

Figure 2(c) shows the PL spectrum of $\mathrm{ZnS}:(\mathrm{Pr}, \mathrm{Mn})$ nanoparticles. It shows both characteristics of $\mathrm{Mn}$-doped (peak on $585 \mathrm{~nm}$ ) and Pr-doped (peaks on 430, 460, 480 and $495 \mathrm{~nm}$ ) $\mathrm{ZnS}$ nanoparticles. The comparison of PL and EL spectra indicates the significant red shift on EL spectra, which is supposed to be the heat effect or the energy transfer to larger nanoparticles[7].
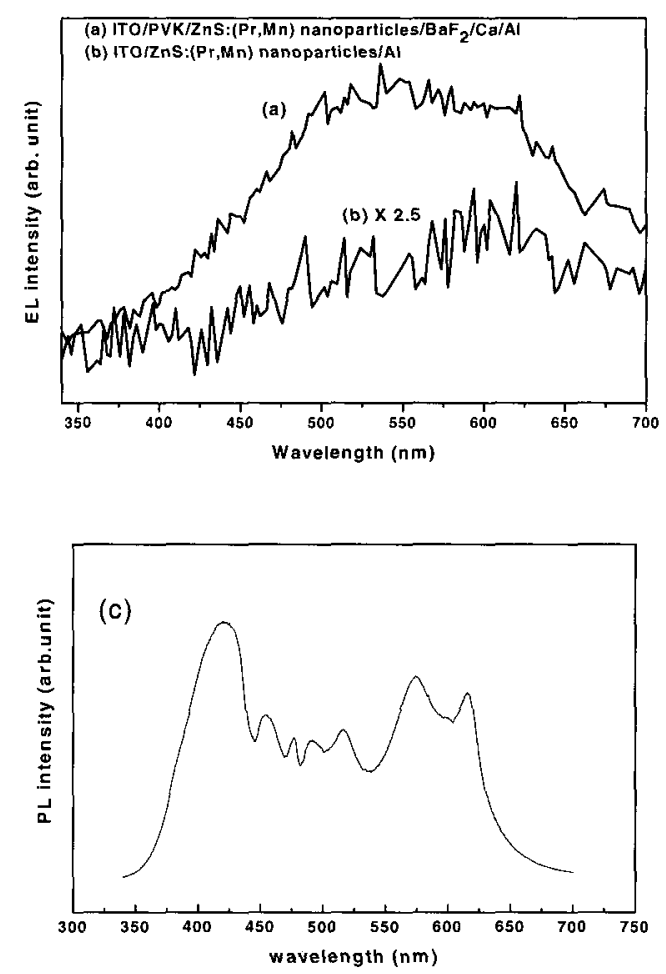

Fig. 2. EL spectra of the device consisting of ITO/ $\mathrm{PVK} / \mathrm{ZnS}$ :(Pr,Mn)nanoparticles/BaF $/ \mathrm{Ca} / \mathrm{Al}$ (a) and ITO/ $\mathrm{ZnS}$ :(Pr,Mn) nanoparticles/Al (b) PL spectrum of $\mathrm{ZnS}$ : $(\mathrm{Pr}, \mathrm{Mn})(\mathrm{c})$. 
Energy diagram of the hybrid EL device consisting of ITO/PVK/ZnS:(Pr,Mn) nanoparticles $/ \mathrm{BaF}_{2} / \mathrm{Ca} / \mathrm{Al}$ is presented in Fig. 3. In this work, organic semiconducting polymer PVK and $\mathrm{BaF}_{2}$ are used as HTL and buffer layer between cathode and nanoparticles, respectively. Due to the large ionization potential of $\mathrm{ZnS}$ :(Pr,Mn) nano-particles, the injection barrier of hole into nanoparticles (the difference between the work function of ITO and ionization potential of $\mathrm{ZnS}$ nanoparticles) is much higher than that of electron (the difference between the work function of $\mathrm{Al}$ and electron affinity of $\mathrm{ZnS}$ :(Pr,Mn) nanoparticles). This leads to the deficiency

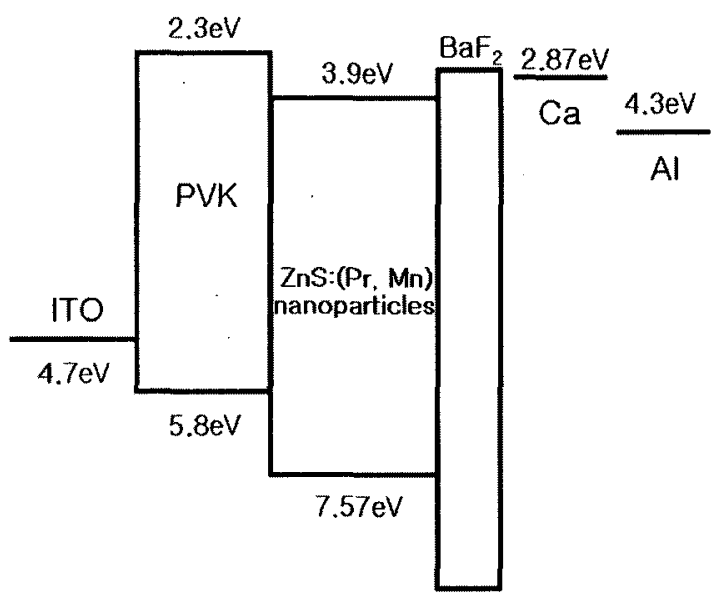

Fig. 3. Schematic drawing of energy diagram for the device consisting of ITO/PVK/ZnS:(Pr,Mn) nanoparticles $/ \mathrm{BaF}_{2} / \mathrm{Ca} / \mathrm{Al}$.

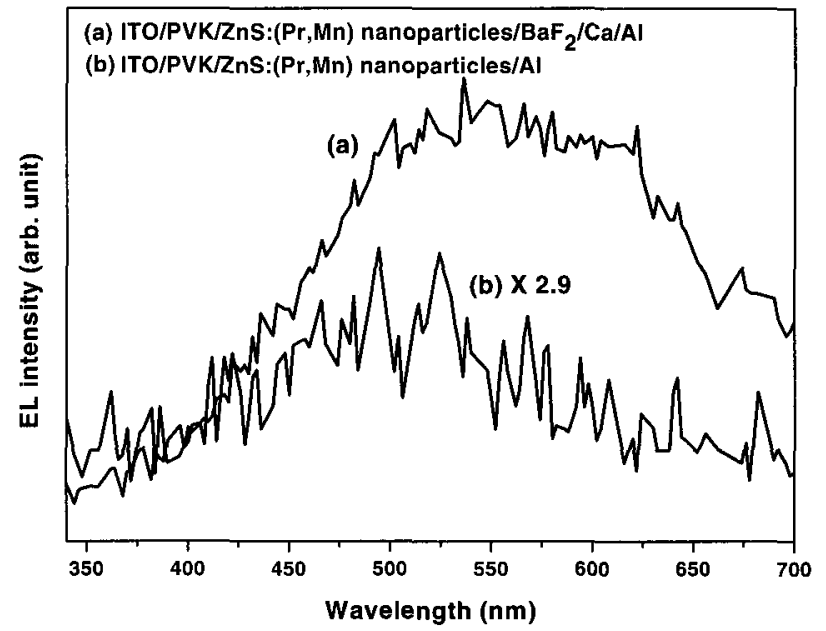

Fig. 4. EL spectra of the device consisting of ITO/ $\mathrm{PVK} / \mathrm{ZnS}:(\mathrm{Pr}, \mathrm{Mn})$ nanoparticles/ $\mathrm{BaF}_{2} / \mathrm{Ca} / \mathrm{Al}$ (a) and ITO/ PVK/ZnS:(Pr,Mn) nanoparticles/Al (b). of holes inside the device in comparison with electron, that is, charge imbalance. Thus, it is necessary to build multilayer structure device for the purpose of improvement in hybrid EL device performance.

Figure 4 shows EL spectra of the devices consisting of ITO/PVK/ZnS:(Pr,Mn) nanoparticles/Al and ITO/PVK/ $\mathrm{ZnS}$ :(Pr,Mn)nanoparticles/BaF $/ \mathrm{Ba}_{2} / \mathrm{Cal}$. When HTL PVK is inserted between ITO and $\mathrm{ZnS}$ :( $\mathrm{Pr}, \mathrm{Mn})$ nanoparticles the EL peak moves to the region ranging from 450 to $520 \mathrm{~nm}$ compared with the case of no PVK. This is due to the shift of emission region from $\mathrm{ZnS}$ :(Pr,Mn) nanoparticles to PVK. Since the transportation of the injected holes is not yet sufficient and the mobility of electrons in the nanoparticles is higher than that of holes in the PVK, it takes much time for electrons to meet with holes and form the excitons, leading to the increase of the distance which electrons must travel until their combination with the holes. Therefore the recombination region is located in the hole transport layer.

On the other hand, the introduction of both HTL(PVK) and buffer layer $\left(\mathrm{BaF}_{2}\right)$ results in the enhancement of EL efficiency compared with the case of the devices consisting of ITO/ZnS:(Pr,Mn) nanoparticles/Al and ITO/PVK/ZnS:(Pr,Mn) nanoparticles/Al The emission spectra range from 500 to $620 \mathrm{~nm}$, showing the fact that the recombination region is formed in the nanoparticle layer. The increase of luminescence efficiency indicates that enhanced charge balance inside the device is attained by the simultaneous use of HTL and buffer layers.

Figure 5 shows the I-V characteristics of as-prepared

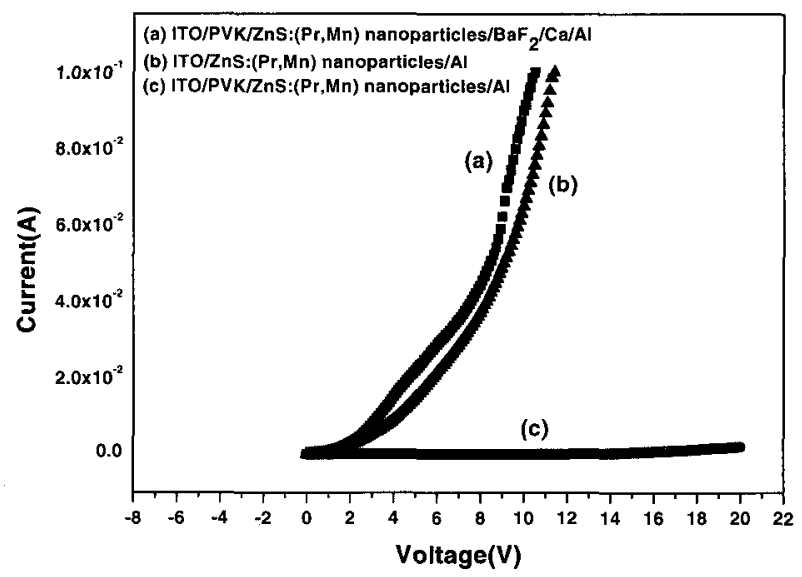

Fig. 5. I-V characteristics of the EL devices: ITO/ PVK/ZnS:(Pr,Mn)nanoparticles/BaF $/ \mathrm{Ca} / \mathrm{Al}$ (a), ITO/ $\mathrm{ZnS}$ :(Pr,Mn)nanoparticles/Al (b), and ITO/PVK/ZnS: (Pr,Mn)nanoparticles/Al (c). 
devices; ITO/ZnS:(Pr,Mn)nanoparticles/Al, ITO/PVK/ $\mathrm{ZnS}:(\mathrm{Pr}, \mathrm{Mn})$ nanoparticles/Al, and ITO/PVK/ZnS:(Pr, $\mathrm{Mn}$ )nanoparticles $/ \mathrm{BaF}_{2} / \mathrm{Ca} / \mathrm{Al}$.

The presence of HTL PVK lowers the current level below that of the single layer device without HTL and buffer layers while the simultaneous insertion of PVK and $\mathrm{BaF}_{2}$ increases the current level above that of the single layer. As seen from the energy diagram, electrons are the dominant carriers inside the devices due to the much higher hole injection barriers. The decrease of current level can be the result of three other cases. The first is that most of the injected electrons recombine with holes due to the increased hole injection. The second is that the flow of the electrons as major carriers is hindered by HTL due to the low mobility in the semiconducting polymer, PVK layer. And the third is the role of PVK as barrier for electron transport. The electron affinity $(2.3 \mathrm{eV})$ of PVK is lower than that (3.9 $\mathrm{eV})$ of $\mathrm{ZnS}$ nanoparticles. So the electrons which move along the energy diagram is confined at the interface between PVK and ZnS nanoparticles due to the energy barrier. Since the EL intensity is not enhanced in the presence of PVK layer, the former can not be applicable to this result. Thus when PVK is present, electrons are accumulated at the interface between PVK and nanoparticles due to the low mobility of electron in the PVK and the energy barrier for electron transport which results from low electron affinity of PVK compared with $\mathrm{ZnS}$ nanoparticles. And it leads to the formation of the internal electric field across the PVK layer. This additional electric field strengthens the externally applied field at the injecting contact between ITO and PVK. Consequently, the hole injection additionally increases together with the increase due to energy-steplike structure. However, when the buffer layer $\mathrm{BaF}_{2}$ is absent, the number of accumulated electrons is not enough to induce a significant internal electric field inside the device. So the additional increase of hole injection due to the internal field is not sufficient.

On the other hand, in the presence of $\mathrm{BaF}_{2}$ buffer layer electron injection is enhanced. This leads to the increase of the number of electrons inside the device, thus the number of accumulated electrons at the PVK interface. At this time the internal electric field enough to result in enhanced hole injection is formed across PVK layer. In a word, the presence of both PVK and $\mathrm{BaF}_{2}$ buffer layer makes it possible to achieve the enhancement effect of hole injection. Thus the charge balance inside the device (consisting of ITO/PVK/ $\mathrm{ZnS}$ :(Pr,Mn) nanoparticles $/ \mathrm{BaF}_{2} / \mathrm{Ca} / \mathrm{Al}$ ) can be improved, which results in the enhancement of EL efficiency.

\section{SUMMARY}

$\mathrm{EL}$ as well as $\mathrm{I}-\mathrm{V}$ characteristics on three devices containing $\mathrm{Pr}$ and $\mathrm{Mn}$ co-doped $\mathrm{ZnS}$ nanoparticles are presented. The insertion of both HTL PVK and buffer layer $\mathrm{BaF}_{2}$ improves device yields; EL efficiency and I$\mathrm{V}$ characteristics. This results from enhanced charge balance inside the device through the enhancement of charge injection, especially holes. The increase of hole injection is due to both the insertion of higher ionization potential material and the formation of sufficiently induced internal field across PVK by the accumulation of the increased electrons at the interface between PVK and nanoparticle layers.

\section{ACKNOWLEDGMENTS}

This work was performed for the Carbon Dioxide Reduction and Sequestration Center, one of the 21st Century Frontier R\&D Programs funded by the Ministry of Science and Technology of Korea (M102KP01000102K1601-01310), and the National Research Program for the 0.1 Terabit Non-Volatile Memory Development sponsored by Korea Ministry of Science \& Technology(MOST).

\section{REFERENCES}

[1] S. Coe-Sullivan, W. Woo, J. S. Steckel, M. Bawendi, and V. Buloric, "Tuning the performance of hybrid organic/inorganic quantum dot light-emitting devices", Organic Electronics, Vol. 4, No. 2-3, p. 123, 2003.

[2] H. Yang, P. H. Holloway, and B. B. Ranta, "Photoluminescent and electroluminescent properties of Mn-doped ZnS nanocrystals", J. Appl. Phys., Vol. 93, No. 1, p. 586, 2003.

[3] R. A. M. Hikmet, D. V. Talapin, and H. Weller, "Study of conduction mechanism and electroluminescence in CdSe/ZnS quantum dot composites" J. Appl. Phys., Vol. 93, No. 6, p. 3509, 2003.

[4] L. Brus, "Electronic wave functions in semiconductor clusters: Experiment and theory", J. Phys. Chem., Vol. 90, No. 12, p. 2555, 1986.

[5] M. C. Schlamp, X. Peng, and A. P. Alivisatos, "Improved efficiencies in light emitting diodes made 
with $\mathrm{CdSe} / \mathrm{CdS}$ core/shell type nanocrystals and a semiconducting polymer", J. Appl. Phys., Vol. 82, No. 11, p. 5837, 1997.

[6] K. Sooklal, B. S. Cullum, S. M. Angel, and C. J. Murphy, "Photophysical properties of $\mathrm{ZnS}$ nanoclusters with spatially localized $\mathrm{Mn}^{2+}$, J. Phys.
Chem., Vol. 100, No. 11, p. 4551, 1996.

[7] S. A. Crooker, J. A. Hollingsworth, S. Tretiak, and V I. Klimov, "Spectrally resolved dynamics of energy transfer in quantum-dot assemblies: Towards engineered energy flows in artificial materials", Phys Rev. Lett., Vol. 89, No. 18, p. 186802-1, 2002. 